\title{
Postpartum Hemophagocytic Lymphohystiocytosis: A New Entity Is Born
}

\author{
Oren Pasvolsky ${ }^{a, b} \quad$ Avi Leader ${ }^{a, b}$ \\ ${ }^{a}$ Institute of Hematology, Davidoff Cancer Center, Rabin Medical Center, Petah Tikva, Israel; ${ }^{b}$ Sackler School of \\ Medicine, Tel Aviv University, Tel Aviv, Israel
}

Hemophagocytic lymphohystiocytosis (HLH) is a rare hyperinflammatory clinical syndrome, provoked either by a primary genetic defect or by a variety of underlying etiologies, including infections, malignancies, and autoimmune diseases. Natural killer (NK) cell and cytotoxic T lymphocyte (CTL) dysfunction plays a key role in HLH pathogenesis. Diagnosis of HLH is supported by meeting at least 5 of 8 clinical, laboratory, and pathological criteria. Therapy aims to suppress the disorderly flames of the immune response, cure the underlying disease, or both. $\mathrm{HLH}$ has been described in many clinical scenarios, amongst a variety of patient populations of all ages. The prognosis of the adult patient with HLH is typically dismal, especially when HLH is secondary to an underlying malignancy. Nonmalignant etiologies may have a more favorable natural history $[1,2]$.

There are several reports on HLH secondary to pregnancy. For example, Yildiz et al. [3] recently described 21 cases of pregnancy-related HLH, 3 of which were diagnosed during the postpartum period. In the current issue of Acta Haematologica Song et al. [4] describe the first case series of patients with postpartum HLH. As expected, the cohort consisted of young female patients in their third decade of life. All patients presented with fever, and

\section{KARGER}

(C) 2018 S. Karger AG, Basel

E-Mail karger@karger.com

www.karger.com/aha onset of HLH was usually during the first few days after giving birth.

During the postpartum period, there is gradual reversal of maternal pregnancy-related physiological changes. Although there is no consensus regarding the exact duration of the postpartum period, most of the maternal physiological systems return to their pre-pregnancy state within 6-8 weeks [5]. Fever in the postpartum period is common, and in most cases the etiology is infectious [6]. Interestingly, all 8 patients in the current report had liver enzyme abnormalities, whereas cytopenia was a less prominent feature, which could further hinder timely diagnosis of HLH during postpartum [4].

The authors propose possible mechanisms contributing to development of postpartum HLH. First, physiological T-cell immunomodulation and suppression of NK cell cytotoxicity during pregnancy might persist during the postpartum. Specifically, levels of NKG2A, an inhibitor of NK cytotoxicity, have been shown to rise during postpartum. Second, it seems that HLH could share some pathogenetic features with preeclampsia, since both have immune dysregulation including shift in T cell subpopulations and increase in inflammatory cytokines, such as TNF- $\alpha$, IL-1 $\beta$, IL-6, IL-10, and IFN- $\gamma$. 
Most patients (5 out of 8 ) in the report by Song had no other underlying etiology for their HLH. This seems to further support the notion that faulty postpartum immunological changes might be enough to push the immune system beyond the ledge to disarray. However, the proximity to delivery in the majority of cases raises the possibility that an unnoticed/subclinical event, such as inflammation or infection during child birth could be to blame, at least in some cases.

Most ( 6 out of 8 ) patients received treatment with the HLH-94/04 protocols, and the same proportion survived (6 out of 8). Compared to pregnancy-related HLH, treatment of HLH after delivery is not limited by safety concerns for the fetus, and patients in this series also received chemotherapy-containing regimens. The 2 patients who died had signs of central nervous system involvement of their HLH, which has previously been shown to be associated with adverse prognosis. In the postpartum subgroup $(n=3)$ of a prior pregnancy-related HLH case series [3], all 3 patients had an underlying disease ( 2 with rheumatologic illness and 1 with an infectious etiology). Two patients achieved complete remission after steroid therapy, while the third had incomplete data on follow-up. Importantly, none of the 11 patients with postpartum HLH in the reported literature $[3,4]$ had an underlying malignancy.
The current report by Song is limited by a small number of patients. However, considering the rarity of HLH, especially in the postpartum setting, this case series adds to our knowledge on conditions associated with HLH. In addition, this report has limited obstetric information, such as delivery method, which is important since the HLH symptoms and signs began close to delivery in most cases. Future studies should provide more detailed information on this aspect.

In conclusion, the report by Song et al. [4] adds valuable information regarding a rare manifestation of a rare disease. Increased awareness to the possibility of HLH during pregnancy as well as the postpartum period is vital for early diagnosis and in order to provide timely therapies. Further studies will hopefully shed light on the pathophysiology of this unique clinical entity and enable better clinical management. Additional observational data could further refine unique characteristics of this newly defined entity.

\section{Disclosure Statement}

The authors have no conflicts of interest to declare.

\section{References}

1 Janka GE, Lehmberg K. Hemophagocytic syndromes-an update. Blood Rev. 2014 Jul; 28(4):135-42.

2 Schram AM, Comstock P, Campo M, Gorovets $\mathrm{D}$, Mullally A, Bodio $\mathrm{K}$, et al. Haemophagocytic lymphohistiocytosis in adults: a multicentre case series over 7 years. Br J Haematol. 2016 Feb;172(3):412-9.
3 Yildiz H, Vandercam B, Thissen X, Komuta M, Lanthier N, Debieve F, et al. Hepatitis during pregnancy: A case of hemophagocytic lymphohistiocytosis. Clin Res Hepatol Gastroenterol. 2018 Jun;42(3):e49-55.

4 Song Y, Wang JS, Wang YN, Wang Z. Hemophagocytic lymphohistiocytosis during postpartum of pregnancy: report of eight cases. Acta Haematol. 2019; in press.
5 Romano M, Cacciatore A, Giordano R, La Rosa B. Postpartum period: three distinct but continuous phases. J Prenat Med. 2010 Apr; 4(2):22-5.

6 Hamadeh G, Dedmon C, Mozley PD. Postpartum fever. Am Fam Physician. 1995 Aug; 52(2):531-8. 